\title{
Culturally congruent mental health research in Africa: Field notes from Ethiopia and Senegal
}

Nicole M Monteiro', Shyngle K Balogun²

${ }^{1}$ Department of Psychology, University of Botswana, Gaborone, Botswana, Address: Private Bag 00775, Gaborone, Botswana

${ }^{2}$ Department of Psychology, Ibadan University, Ibadan, Nigeria

\begin{abstract}
In this article we discuss culturally congruent methods for conducting mental health research in Africa, with a focus on field work in Ethiopia and Senegal. Our goal is to emphasize the centrality of culture to carrying out mental health research and to present community-participatory and qualitative approaches as frameworks for mental health research in Africa. We use field research examples from Ethiopia and Senegal to highlight key cultural considerations, including: obtaining community buy-in, connecting with local stakeholders, determining appropriate research questions, collaboration in developing questionnaires, working with interpreters, facilitating useful disclosure of findings and interpreting meaning from research data. Finally, we make the case for the richness of qualitative field research as a way to examine complex psychological and psychiatric topics.
\end{abstract}

Keywords: Africa, sub-Saharan; cultural competence; mental health; qualitative research

Received date: 2 -12-2013

Accepted date: $30-01-2014$

doi: http://dx.doi.org/10.4172/Psychiatry. 1000108

\section{Introduction}

There is increased awareness and recognition of the importance of conducting culturally relevant mental health research in Africa. Some reasons include interest in traditional healing practices and indigenous concepts of mental illness, efforts to address disparities in mental health care worldwide, and the need to broaden the scope of cross-cultural research to enrich basic knowledge in psychology and the behavioural sciences. However, conducting culturally relevant mental health field research can be burdened with a number of challenges such as resource/logistical constraints, ethical problems and cultural misunderstanding. ${ }^{1}$ For example, there may be limited materials and support for local researchers, bureaucratic challenges for foreign investigators, and most strikingly, unique considerations regarding ethics and interpretation of meaning given the cultural context. Some of these challenges result from: the stigma associated with investigating sensitive mental health topics; language barriers; and difficulty convincing funders and stakeholders of the importance of mental health research, especially given the prevalence of other serious health problems. ${ }^{2}$ There is a unique set of research skills, competencies and culturally congruent research methods that can address some of the aforementioned challenges. We posit that the premise underlying these skills and approaches is attention to the impact of culture in all phases of the research process.

The increase in multi-disciplinary research throughout Africa has prompted a critique of existing methods and procedures. Scholars have questioned whether

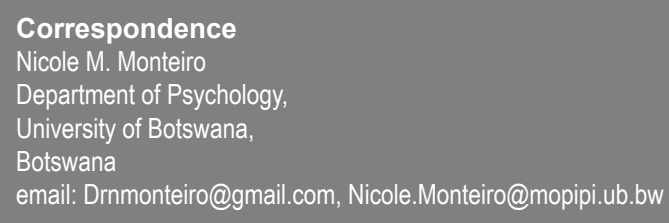

traditional "objective" methods are the most effective for obtaining valid, reliable and accessible research data and information from diverse populations. ${ }^{3}$ One theoretical perspective questions whether assumptions underlying the paradigms, approaches and methodology used in largescale quantitative and population research are appropriate for all settings, groups and research inquiries. The question becomes, are the methods used in broad survey or epidemiological analysis adequate to address the ways that culture impacts research procedures and interpretation of results?

\section{Centrality of culture}

Culture is defined as enduring patterns of behaviour and modes of relating within a community or society that are passed on inter-generationally and include, but are not limited to: language; modes of production; expressed values, attitudes, and beliefs; rituals related to birth, death and other life milestones; food and eating patterns; art, music and dance; styles of dress and other expressions of ethnic identity. ${ }^{4}$ Culture also encompasses deeper, latent processes that are less easily identified, such as worldview, epistemology, theological perspectives and implicit ethics, ideals and morals. Adequate treatment of culture in research must attend to these subtle intangible processes as well as the more easily identified manifestations of cultural patterns, especially when carrying out research in a culture different from one's own.

Some mental health researchers have highlighted the importance of considering and attending to culture at every stage of research, from conceptualization and formulation of research questions, to implementation of methods and procedures, and interpretation of findings. ${ }^{5}$ The centrality of culture is particularly salient for researchers investigating mental health issues cross-culturally that are sensitive or are not well recognized in the society, such as sexual behaviour, domestic violence, substance use, personal experiences 
with mental illness, family values and others. Hughs and colleagues underscore the integral role that culture assumes throughout the entire research process, including formulation of research questions, operationalization of concepts, and development of research design. ${ }^{5}$ They provide guidelines for increasing awareness and for exploring and accommodating cultural assumptions and beliefs in social science research methodology. The bottom line is that researchers should be culturally alert when carrying out research in cultures different from Western societies where much of the discourse on appropriate methods and procedures is centred.

\section{Frameworks for Mental Health Research in Africa}

We have found two frameworks particularly useful in developing culturally congruent mental health research. Best practices for conducting research with under-served communities include community participatory research and qualitative research.

The community-participatory research approach seeks to "increase the value of studies for both researchers and communities participating". ${ }^{6}$ It stems from a perspective that the most accurate useful information is acquired when there is a collaborative relationship with and strong participation from the community. There is also the assumption that embedded in the research design is the goal of providing some outcome benefit to the community.

Qualitative research methods are typically used to investigate populations and questions that do not lend themselves well to large-scale quantitative investigation or for topics that require more nuanced and rich perspectives from which to make inferences or draw conclusions. ${ }^{7}$ Researchers have increasingly focused on eclectic or mixed-methods, which integrate quantitative and qualitative techniques, for mental health field research. This methodology has gained acceptance as a way to more fully capture phenomenal and relational information about a community. This approach is important because within the global health filed mental health research is expected to be applicable to and result in viable impact for communities.

Some disciplines, such as community and cross-cultural psychology, have utilized a combination of qualitative and quantitative approaches to address cultural variables that might otherwise be missed in traditional survey research. ${ }^{8}$ Some of the identified strengths of qualitative approaches include richness, detailed information, and improved interpretation of constructs. $^{9}$

Factors such as obtaining buy-in from marginalized communities, encouraging participation in research that is relevant to the target community, developing culturally congruent research questions and procedures, and liaising with government and official ministerial and other community stakeholders are key to developing culturally congruent methodology. These components of research procedure are not always formally taught, but have proven to be meaningful with some African populations. For example, groups with a communal cultural orientation may require additional outreach activities or consent procedures besides those targeted only to individual research participants.

Traditional methods are incongruent when they fail to establish culture and the related social context as a central variable in developing research. Our goal in this paper is to address that problem by identifying some of the culturally congruent research skills and methods that are appropriate for mental health field research in Africa and to illustrate field examples from the first author's research experiences in Ethiopia and Senegal. The examples describe challenges of research in the field and identify competencies that help to address those challenges.

\section{Field Case Notes}

\section{Researcher Background}

The first author is trained as a clinical psychologist and researcher. Her background and field experience include extensive qualitative research in Ethiopia and rural Senegal and survey and naturalistic observational research in Botswana, as well as applied clinical work and case study observations in parts of the Caribbean, South America and the Middle East. The focus of much of her research and clinical work has been the practical integration of culturally nuanced methods and a critique of approaches that do not adequately address culture.

The research that was conducted in Ethiopia was a dissertation study that included a qualitative exploratory examination of the society's conceptualization of mental illness. The aim was to examine the perceptions of mental illness in both rural and urban regions of the country. Specifically, healthcare workers, traditional healers and laypersons were surveyed about their attitudes, beliefs and practices regarding mental illness. Semi-structured interviews were used to discuss definitions, symptoms, causation and treatment practices related to mental health and illness. Respondents were asked about disorders described as depression, anxiety and psychosis, as well as their general understanding of mental illness. The study was important because while there is a body of literature focused on epidemiological trends and diagnostic prevalence in global mental health, there is also a need to enrich the field with descriptive accounts of what mental illness looks like "on the ground," obtained using culturally congruent and scientific procedures.

In Senegal, the first author has also been engaged in community mental health research, exploring different aspects of how health and illness are perceived and addressed by the community in rural villages in Southeastern Senegal (in the Kedougou region). Some of these researches were carried out as part of a global mental health training program that gave advanced graduate students from the West exposure to the culture and health care system in Senegal. The projects were conducted in collaboration with the local health district and a local NGO. The first author supervised students' research projects which included brief studies on the treatment of the severely mentally ill, perceptions of domestic violence, HIV awareness, childhood development, geriatric mental health, and cultural competency among health workers. In Ethiopia and Senegal, ethics clearance was obtained from the ministry of health and the district health officer, respectively. Studies from the Ethiopia research were published in 2013 (See ${ }^{10,11}$ ). Research manuscripts from the Senegal research are currently under preparation.

The emphasis in each of these projects was the usefulness of semi-structured one-on-one and focus group interviews to obtain relevant and sensitive information about attitudes, perceptions and beliefs about health and illness. In this case, the focus was on mental health, specifically issues around definition, identification, treatment and stigma. However, 
the qualitative, community-oriented methodology adopted here has relevance for multiple areas and fields within mental health and broader social science research. This approach can be seen as a cultural meta-process that focuses on the consideration of cultural dynamics throughout the course of the research.

In the following field descriptions some of the challenges and unique opportunities inherent in developing and applying the aforementioned culturally-congruent qualitative methodology for mental health research in Africa are presented. Attention is drawn to the unique skill set, knowledge and competencies that are required to effectively carry out culturally grounded mental health research.

\section{Field Methods}

The common thread among these projects is the emphasis on culture from inception to completion and the development of methods to ensure community participation and culturally congruent and valid results. Our recommended approach to mental health field research in Africa assumes the centrality and a priori importance of culture in all conceptual and procedural stages of investigation. The following approaches and strategies are a part of a broad research process, which includes community-participatory and/or qualitative methods. The methods and procedures we describe are referred to as culturally anchored mental health research strategies.

Community buy-in: This perhaps is the most fundamental step in the research process. Essentially, it entails establishing rapport and trust with the community to facilitate the on-going, collaborative working relationship that will define the remainder of the research process. Buyin is as much about building confidence in your methods as it is about establishing a relationship with the community. It is a way of establishing face validity for the evaluative, research or assessment procedures being used. Also, the rapport needed to gain the participants' confidence is often developed through surrogates or individuals who already have a track record of service and trust in the community. In the case of our research team in Senegal, it was the medical clinic staff who had been working in the community for several years.

One of the research challenges in Ethiopia was obtaining the necessary research permit from the appropriate ministry. Criteria for obtaining the permit included demonstrating the importance of the research for the community and evidence that the local population would be open to participating in the research. Our research group decided to make contact with key leaders and stakeholders in the community who could advise about how to engage the population and give feedback on what issues related to our research seemed most salient to the community. These efforts were not necessarily aimed at changing the actual research, but gauging how to clearly communicate its importance and relevance. While this is a requirement of all community based research, we stressed community buy-in in these projects as an illustration of the cultural importance of seeking permission, literally and also metaphorically.

Making connections with stakeholders: Stakeholders are the key members of the society and community who have significant influence on the attitudes, opinions and behaviour of the broader community. In many traditional societies and communal villages, at least one stakeholder, such as the chief or community spokesperson, should sanction or give their approval of the research activities to help achieve the buy-in referenced above. For example, for the research projects in Senegal, the team needed to approach stakeholders at various levels of formality, including representatives from the ministry, officials from the health district, political officers (such as la prefecture of the town) and the traditional village chief. These steps sent the message that we respected the implicit and explicit protocol of the community and larger government entities and that we valued the boundaries established by said protocol. It was also a way of bestowing a level of cultural legitimacy on the project.

Determining appropriate questions with the community: Attending to cultural appropriateness includes research on question conceptualization, measure development and piloting. As part of conceptualizing the research aims and objectives, the researcher is tasked with thinking about larger systems and influences beyond the specific question. This means that when thinking about the approaches to take in examining mental illness prevalence, the investigator is charged with exploring the cultural worldview of wellness and illness, as well as communication patterns and subtle manners of conveying information that impact how a study participant would respond to certain questions. This step is crucial in questionnaire and measure development because it is at the foundation of how one develops, selects and words questions. It also shapes how observations and semi- and unstructured interviews are formulated and interpreted. Both in Ethiopia and Senegal, we reviewed initial interview questions with cultural experts in the community and then piloted them with community members in small focus groups. The cultural experts were individuals who were identified as having an understanding and knowledge of traditions, values and attitudes in the society.

Crafting interview and focus group questionnaires: This process entails further specification and refinement of the information obtained during the conceptualization stage. Ideally, the questionnaires should go through an informal and/or formal piloting process whereby direct feedback from community members might result in clarification of definitions and terms, improvement of examples used and revision of the interview format. As part of the Senegal research, we determined that certain idioms of distress were better suited for use with a particular ethnic group in the region where the study was conducted, even though a slightly different idiom was used in another village. Also, the research team discovered that some topics, such as women discussing their perceptions of the impact of domestic violence on emotional health, were better explored in group format or by giving respondents story vignettes to which they could respond orally.

Working with interpreters: Use of translators is fraught with the potential pitfall of misinterpretation, but also presents opportunities to obtain rich information from populations whose literal and broader symbolic voices are often underrepresented in research. Often, because of the linguistic diversity throughout Africa, even if a researcher is from the country where he or she is conducting research, language may be a barrier, as interviews may be conducted by individuals who may be of a different ethno-linguistic group than the target community. Problems include many of the obvious concerns such as incorrect translation, untrained 
or poorly trained interpreters, lack of parallel or practical equivalents, and conceptual problems. Our field research in Ethiopia entailed a detailed process of translating questions from English to Amharic, translating back to English and then piloting and modifying the questionnaire for validity. With focus groups and less structured oral interviews, it was important to audio record as well as to transcribe responses at the same time. This procedure allowed for a back and forth between the research team and the participants at the time of the interview, and it also ensured the reliability of the data by having audio that could be reviewed and translated again later. In Senegal, we observed that as part of responding to focus group questions participants would tell stories to illustrate their points or opinions. It was important for interpreters to be aware of this tendency and not interrupt respondents in the middle of their stories in order to translate. That was the purpose of the audio - to have a recording against which to check the initial translation.

Facilitating useful disclosure: This component of the overall cultural research strategy is about collaborative exchange with the community. Whether community stakeholders, community representatives or research participants, the population is best served by receiving feedback from the research team about results, recommendations and other information related to findings. Of course, this disclosure has to be directed by ethical guidelines that ensure participants understand the information they are receiving and that it is relayed in a clear, practical manner that allows them to utilize and incorporate the information and recommendations. For example, after our research training group in Senegal completed each of the individual projects they wrote a summary report for the health clinic staff, many of whom had participated in the research as respondents or who ran programs that were directly related to the research that was conducted. Our purpose in providing the summaries was to provide transparent feedback that could be utilized on the ground in a practical manner that was quite different from the somewhat delayed and often less practical impact of a published journal article.

Interpreting meaning: The interpretation step could be considered one of the most important. That is because a wealth of pertinent information can be obtained through precise cultural understanding or, conversely, missed or overlooked as a result of misunderstanding or misinterpretation. The aim of developing the appropriate methodology is to be able to collect data that are clean enough to further analyse and interpret. However, when conducting mental health field research, there are potentially numerous challenges to drawing useful conclusions and accurate interpretations from the findings. There is always the issue of the cultural and philosophical assumptions that guide researchers' theoretical underpinnings and that either explicitly or implicitly shape the interpretations and conclusions. There is a body of research which looks at the interplay of researcher values and participant experience in participatory and qualitative research. 12,13 Recognizing that researchers are a possible source of research bias is a necessary effort in any attempt to address this inherent bias.

Generalizability and applicability to larger populations: The advantage of small-scale qualitative research is that is provides a rich set of data with nuance, complexity and specific meaning. This type of information can be useful for policy planning. In addition to contributing to general scientific and theoretical knowledge, culturally-informed and qualitative research can fill in some of the gaps and provide the on-the-ground perspective needed for multifaceted policy planning which requires a practical view of the problem at hand. In addition, the type of research described here often acts as a cultural bellwether for pertinent dynamics and upcoming changes within the community. Yet another benefit of culturally-informed qualitative research is that it acts as an excellent adjunct to quantitative analysis by providing timely indicators of emerging issues within the community that would be difficult to discern with surveys.

\section{Conclusion}

Despite some of the conceptual and practical challenges of carrying out mental health field research in African settings, the opportunities and necessity of doing so have numerous benefits. Enriching the knowledge and understanding of universal and culture-specific mental health conditions, broadening the scope of cross-cultural and communitybased research, improving and refining research methodology and theory, and reducing marginalization and stigma toward mental health are among them. In particular, it is paramount that researchers appreciate the process of recognizing the importance of culture and employing it to conceptualize and interpret research, especially on sensitive topics. This process for investigators should include multiple approaches to developing the research agenda and disseminating results so that both are constructive and add value to the community. Qualitative field research provides a rich avenue for achieving these goals and should be a standard component of mental health research programs that target complex issues.

\section{Declaration of Relative Contribution}

Author 1 conceptualized the paper, conducted original studies, conducted literature search and wrote the manuscript.

Author 2 contributed to structure of paper, conducted literature review, revised final draft.

\section{References}

1. Fernando GA. The roads less traveled: Mapping some pathways on the global mental health research roadmap. Transcultural Psychiatry 2012; 49: 396-417.

2. Alem A, Kebede D. Conducting psychiatric research in the developing world: Challenges and rewards. The British Journal of Psychiatry 2003; 182: 185-187.

3. Zalenga $S$. The use of empiricist and narrative methods in comparative social science research: Lessons and insights for third world studies and research. Journal of Third World Studies 201 1; 28: 107-141.

4. Erchak GM. The Anthropology of Self and Behavior. New Brunswick, NJ. Rutgers University Press, 1992.

5. Hughes D, Seidman E, Williams N. Cultural phenomena and the research enterprise: Toward a culturally anchored methodology. American Journal of Community Psychology 1993; 21: 687-703.

6. AHRQ. Activities Using Community-Based Participatory Research to Address 2009; Agency for Healthcare Research and Quality: Rockville, $M D$.

7. Maton KI.A Bridge between cultures: linked ethnographic- 
empirical methodology for culture anchored research. American Journal of Community Psychology 1993; 21 : 747-773.

8. Hines, AM. Linking qualitative and quantitative methods in cross-cultural survey research: techniques from cognitive science. American Journal of Community Psychology 1993; $21:$ 729-746.

9. Johnson BR, Onwuegbuzie A. Mixed methods research: $A$ research paradigm whose time has come. Educational Researcher 2004; 33: 14-26.

10. Monteiro NM, Balogun SK. Perceptions of mental illness in Ethiopia: A profile of attitudes, beliefs and practices among community members, healthcare workers and traditional healers. International Journal of Culture and Mental Health 2013; 1-14.
11. Monteiro NM, Balogun SK. Urban and rural perceptions of mental illness in Ethiopia. IOSR Journal of Humanities and Social Sciences 2013; 8: 43-51.

12. Al-Natour $R \mathrm{~J}$. The impact of the researcher on the researched. M/C Journal 2011; 14 (6): Retrieved from http://journal.media-culture.org.au/index.php/ mcjournal/article/viewArticle/428.

13. Ganga D, Scott, S. Cultural "insiders" and the issue of positionality in qualitative migration research: Moving "across" and moving "along" researcher-participant divides. Forum Qualitative Research 2006; 7(3): Retrieved from http://www.qualitative research.net/index.php/fqs/ article/view/134/289. 